\title{
Progressive macular hypomelanosis among Egyptian patients: a clinicopathological study
}

\author{
Mohamed Khaled Selim, M.D. ${ }^{1}$, El-Shahat Farag Ahmed, M.D. ${ }^{1}$, \\ Mamdouh Morsy Abdelgawad, M.D. ${ }^{1}$, Mohammed Fawzy El-Kamel, M.D. ${ }^{1}$
}

${ }^{1}$ Department of Dermatology, Andrology and STDs, Mansoura University, Egypt

Key words: progressive macular hypomelanosis

Citation: Selim MK, Ahmed EF, Abdelgawad MM, El-Kamel MF. Progressive macular hypomelanosis among Egyptian patients: a clinicopathological study. Dermatol Pract Concept 2011;1(1):3. http://dx.doi.org/10.5826/dpc.0101a03.

Editor: Harald Kittler, M.D.

Received: October 1, 2010; Accepted: February 11, 2011; Published: October 31, 2011

Copyright: $@ 2011$ Selim et al. This is an open-access article distributed under the terms of the Creative Commons Attribution License, which permits unrestricted use, distribution, and reproduction in any medium, provided the original author and source are credited.

Funding: None.

Competing interests: The authors have no conflicts of interest to disclose.

All authors have contributed significantly to this publication.

Corresponding author: Mohamed Khaled Selim, M.D., Department of Dermatology, Mansoura University, 60 El Gomhoria Street, Mansoura, 35516 Dakahlia, Egypt. Email: mkhaled4@yahoo.com.

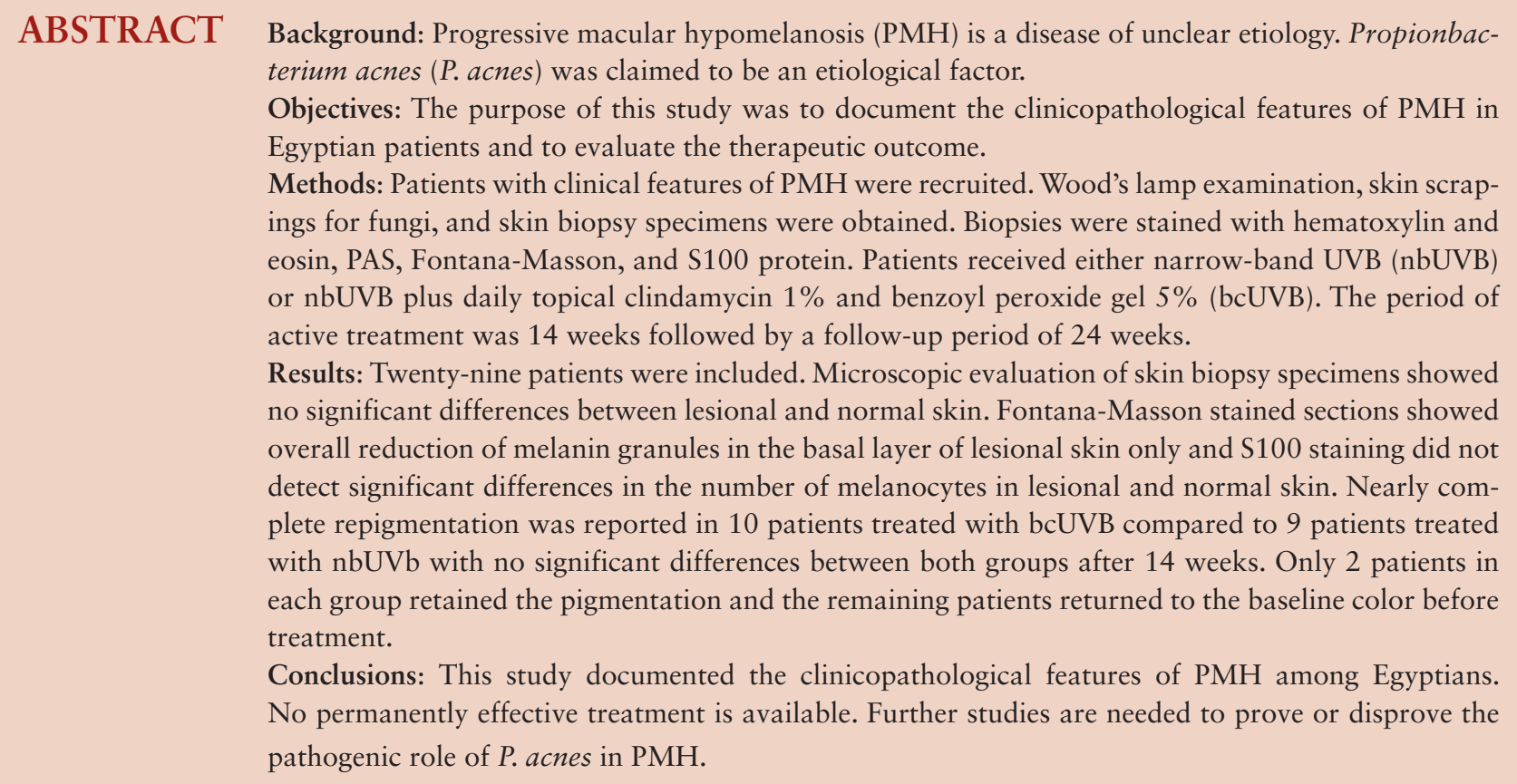




\section{Introduction}

Progressive macular hypomelanosis $(\mathrm{PMH})$ is not a common skin disorder but it may be only rarely recognized. It is often mistaken for pityriasis versicolor and pityriasis alba. Various authors have written about similar skin disorders, referring to them by different names, but we believe that all these similar disorders are part of the same entity. The current term "progressive macular hypomelanosis" was coined by Guillet et al [1] following a study in the French West Indies concerning this condition in Black adults of mixed ethnic origin. Subsequently, additional cases were reported from other parts of the world [2-4].

$\mathrm{PMH}$ is characterized by ill-defined nummular, non-scaly hypopigmented spots on the trunk, often confluent in and around the midline, and rarely extending to the proximal extremities and neck/head region. There is normal sensation without any history of itching, pain, or preceding infection, trauma or inflammation [1-5]. PMH has a worldwide distribution; however, it is more often identified in Black people living in or originating from tropical countries [1-5]. It is also more often seen in young females $[1,2,4,5]$. However, one study reported a higher frequency in males [3].

The natural history of $\mathrm{PMH}$ is stable disease or perhaps slow progression over decades, with spontaneous disappearance after mid-life [1, 2, 4]. However, others have reported that spontaneous regression does not occur and that the hypomelanosis remains stable for many years $[3,5]$.

Extensive pityriasis alba is probably identical with $\mathrm{PMH}$, and discontinuation of use of the former term was suggested on the grounds that extensive pityriasis alba is histologically and clinically different from classical pityriasis alba, which is basically an eczematous type of disorder [6].

$\mathrm{PMH}$ is characterized histologically by diminished pigment in the epidermis and a normal-looking dermis [1, 4]. Electron microscopy shows a shift from large melanosomes in normal-looking skin to small aggregated, membrane- bound melanosomes in hypopigmented skin [1, 4]. PMH should be differentiated from other disorders with hypopigmentation on the trunk, such as pityriasis versicolor, mycosis fungoides, vitiligo, leprosy, pityriasis alba, and postinflammatory hypopigmentation [1-6].

The etiology of this condition is uncertain, although Westerhof et al recently incriminated Propionobacterium acnes [5]. PMH appears to progress to some extent over months to years and then becomes relatively static. In patients with dark skin types, PMH may be distressing because the contrast between normal skin and hypopigmented macules makes the lesions appear more prominent and the patients feel socially awkward [1-4].

No consistently effective therapy is known, but lesions may spontaneously be resolved. However, there are few reports of repigmentation after narrow band UVB [7, 8], combination of sunlight and oral tetracycline (case report) [9], combination of UVA1 plus topical clindamycin and benzoyl peroxide (45 patients) [10], or combination of topical benzoyl peroxide and oral doxycycline (case report) [11].

The purpose of this study was to document the clinicopathological features of $\mathrm{PMH}$ in Egyptian patients, and to evaluate the therapeutic outcome.

\section{Material and methods}

Patients, who were at least 16 years of age, presenting to Dermatology Clinic of Mansoura University Hospital, Mansoura, Egypt with acquired, hypopigmented, discrete or confluent macules larger than $1 \mathrm{~cm}$ in diameter, with normal sensation, consistent with the clinical features of PMH described by Gullet et al $[1,2]$ were selected. Thirty-five patients fulfilling these criteria were enrolled in this study during the period of February 2006 to January 2010. Patients were excluded if their hypopigmentation was associated with or the result of a specific disease at the site, such as pityriasis versicolor,

Table 1: Clinical characteristics

Characteristics

\begin{tabular}{ll}
\hline Female & $22(76 \%)$ \\
Male & $7(24 \%)$ \\
Age, years (mean \pm SD) & $20.7 \pm 9.1$ \\
Duration, years (mean \pm SD) & $7 \pm 2.1$ \\
Former treatment & \\
PUVA & 1 \\
UVB & 2 \\
Antimycotics & 19 \\
No treatment & 7
\end{tabular}


hypopigmented macules of leprosy, hypopigmented mycosis fungoides, idiopathic guttate hypomelanosis, eczema, psoriasis, pityriasis lichenoides chronica, pityriasis rosea, injury, infection, or congenital disorders of pigmentation, such as piebaldism, nevus depigmentosus, ash leaf macules of tuberous sclerosis, pityriasis alba, and vitiligo. Patients were also excluded if they had positive potassium hydroxide test results; were sensitive to any of the study medication ingredients or sunlight; were treated with chemical peeling or other treatments that could cause scaling of the trunk; or were pregnant or lactating. In addition, any previous treatment for PMH or any antibacterial treatment (both local and systemic) had to be stopped at least three months before study entry. Details of the history and examination were recorded. Patients were evaluated at eight-week intervals for 24 weeks after therapy. Photographs taken at the first visit were compared with those obtained during follow-up evaluations. Informed written consent was obtained from every patient or his guardian.

We obtained two $4 \mathrm{~mm}$ punch biopsy specimens from comparable anatomic sites of lesional and normal-appearing skin. Hematoxylin and eosin staining and periodic acid Schiff staining were performed. Fontana-Masson stain for melanin was performed as well as immunohistochemical stain for S100 protein. Potassium hydroxide (KOH) examination of epidermal scrapings from hypopigmented lesions was performed. Wood's lamp examination in a completely dark room was also done.

The patients were divided into two treatment groups. The first group received narrow-band UVB (nbUVB) treatment (starting dose $100 \mathrm{~mJ} / \mathrm{cm}^{2}$, and increased dose by $50 \mathrm{~mJ} / \mathrm{cm}^{2}$ ) twice weekly for 28 sessions. The other group received the same regimen of first group (nbUVB treatment) plus daily topical clindamycin solution $1 \%$ in the morning and benzoyl peroxide gel $5 \%$ in the evening (bcUVB group). The period of active treatment in both groups was 14 weeks followed by a follow-up treatment-free period for 24 weeks.

Subjective assessment of treatment result (repigmentation) was assessed by comparing pre-treatment and posttreatment photos. Images of the trunk were taken at each visit. Conditions were standardized throughout the study, including the same camera, lighting conditions, and distance between camera and patient, and the same external dermatologist for color assessment. The assessing dermatologist who was blinded for the assigned treatment independently scored treatment success by comparing photos before treatment with photos at the end of the active treatment period, i.e., after 14 weeks, and photos at the end of follow-up treatment free period of another 24 weeks (38 weeks after start of active treatment). Repigmentation relative to the baseline situation was scored on a 3-point scale: $++=$ total repigmentation, $+=$ moderate repigmentation, $0=$ no change .

\section{Statistical analysis}

The subjective assessment scores of the patients' dermatologists and assistants were analyzed by comparing the scores of both treatment groups.

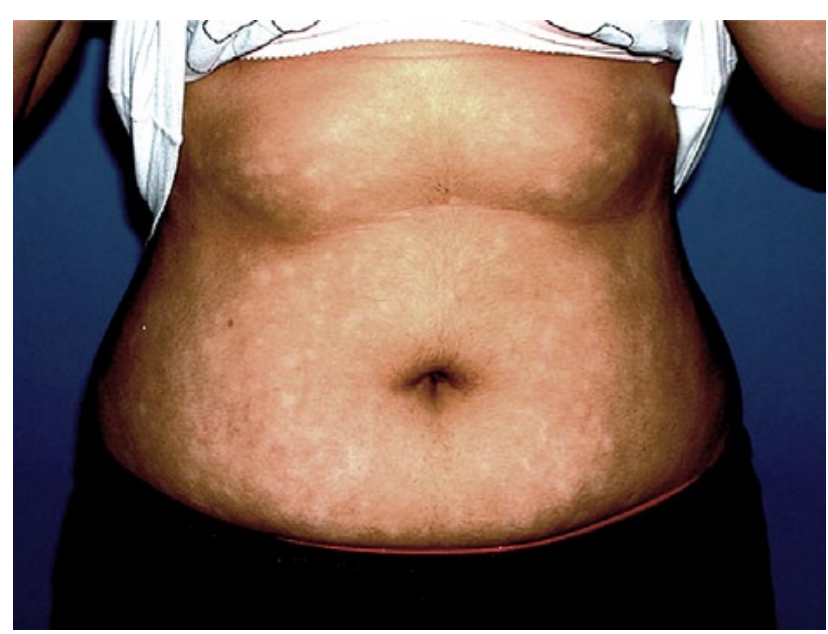

Figure 1. Non-scaly, ill-defined hypopigmented, discrete macules on the abdomen.

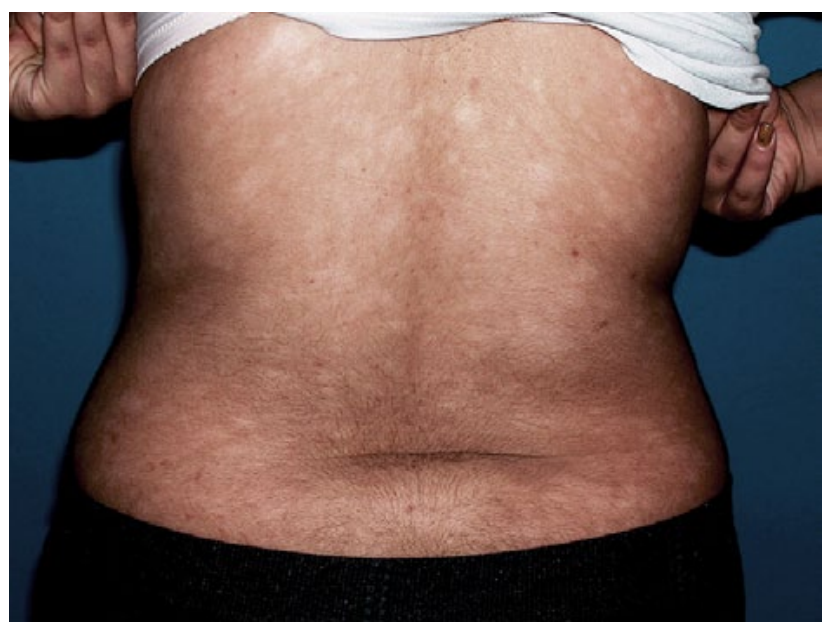

Figure 2. Typical lesions on the back.

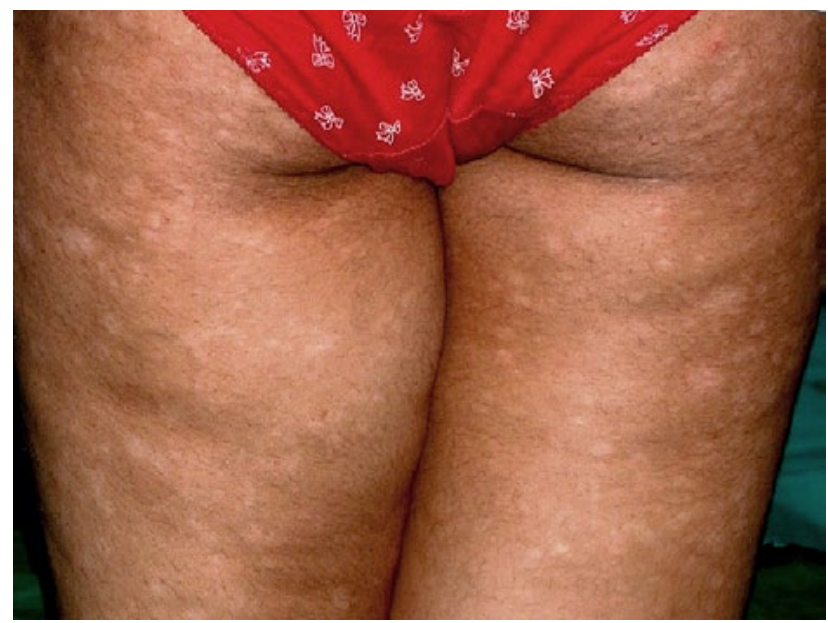

Figure 3. Typical lesions on the buttocks and thighs. 


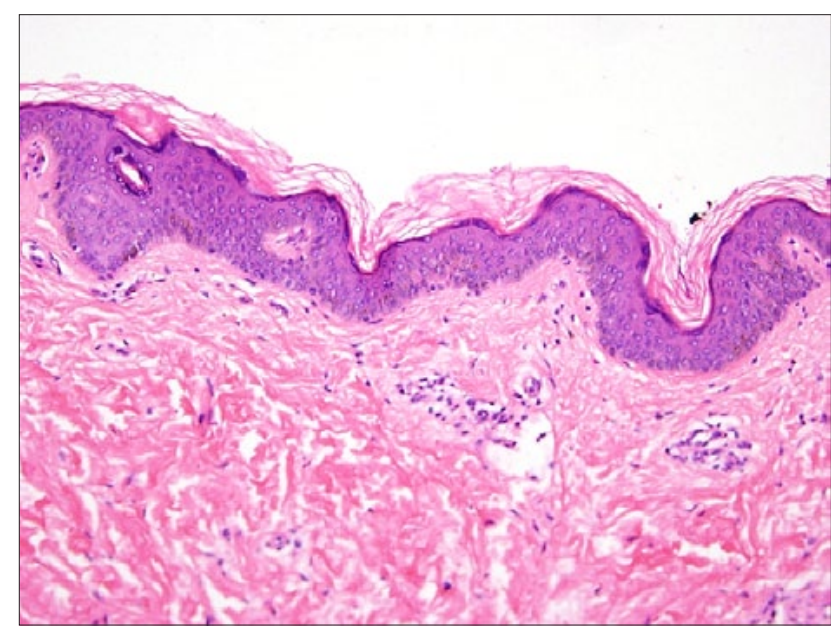

Figure 4. Biopsy from the hypopigmented skin lesion shows decrease in the pigment intensity in the epidermis (hematoxylin \& eosin $[\mathrm{H} \&$ E], 20x).

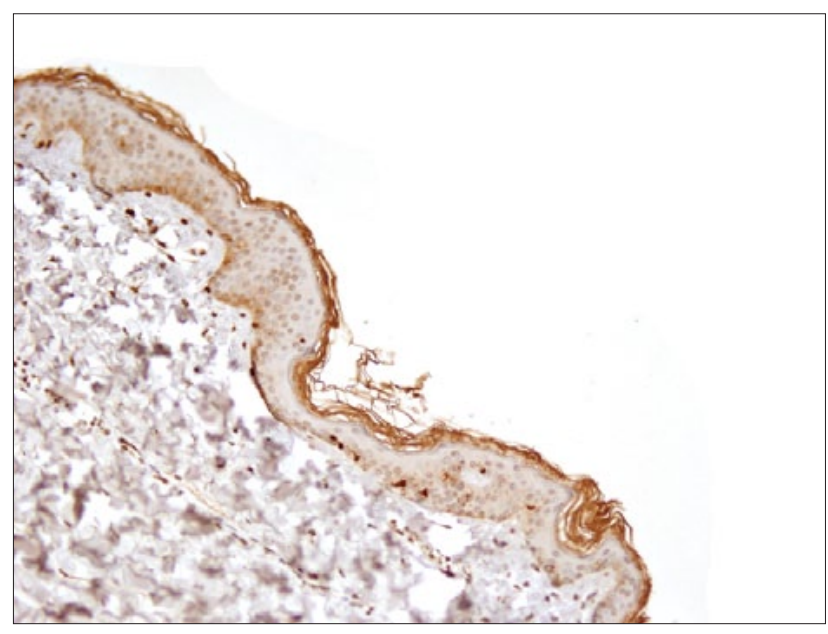

Figure 6. Lesional hypopigmented skin: S100 stained section shows normally intact melanocytes in the basal cell layer like those found in normal-appearing skin biopsy in Figure 7 (S100, 20x).

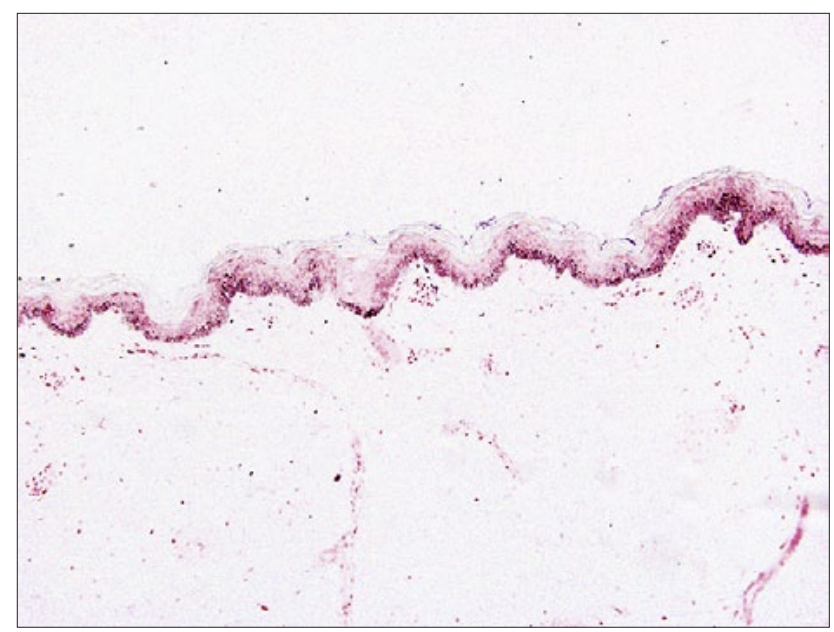

Figure 8. Lesional hypopigmented skin biopsy: Fontana-Masson stained section shows reduction of melanin granules in the basal cell layer of the hypopigmented skin compared with normal pigment of the adjacent normal-appearing skin in Figure 9 (Fontana-Masson, 10x).

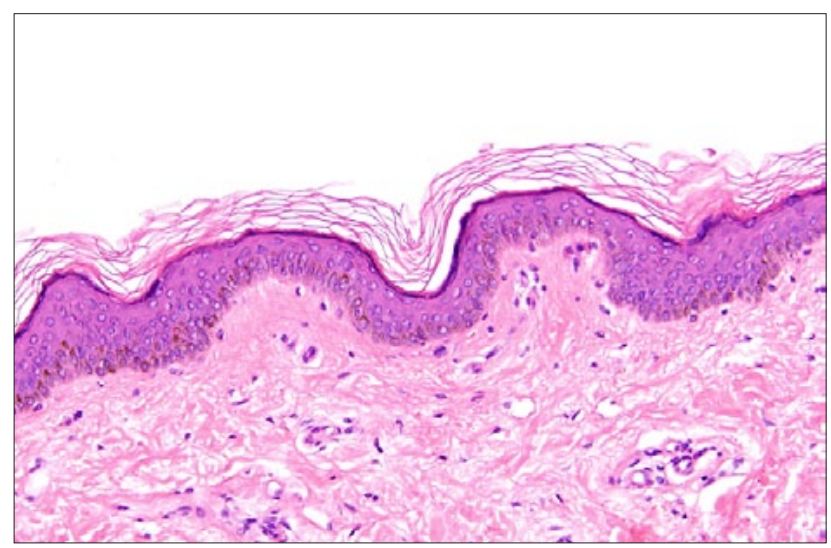

Figure 5. Biopsy from normal-appearing skin shows normal pigment intensity in the epidermis ( $\mathrm{H} \& \mathrm{E}, 20 \mathrm{x})$.

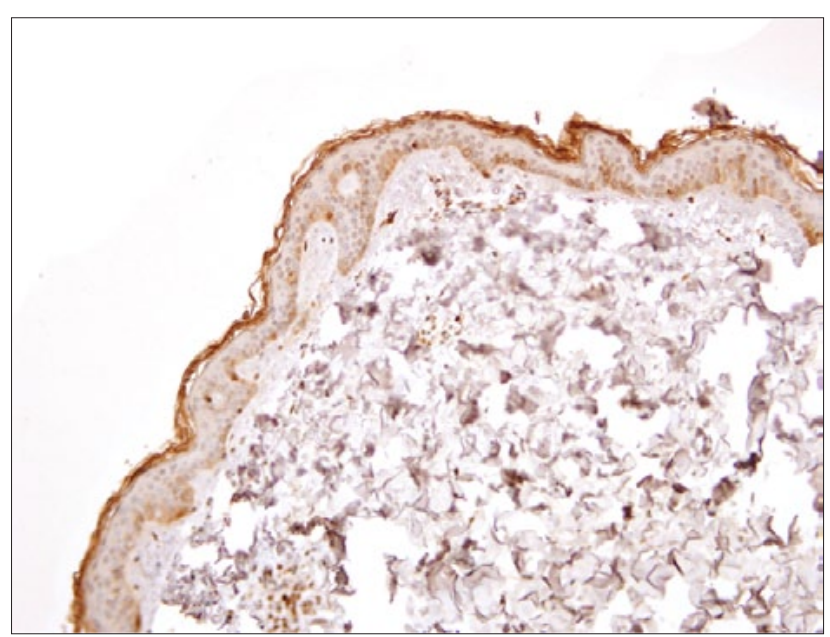

Figure 7. Normal skin: S100 stained section shows no difference in the number of melanocytes in the normal-appearing skin and the hypopigmented skin in Figure 6 (S100, 20x).

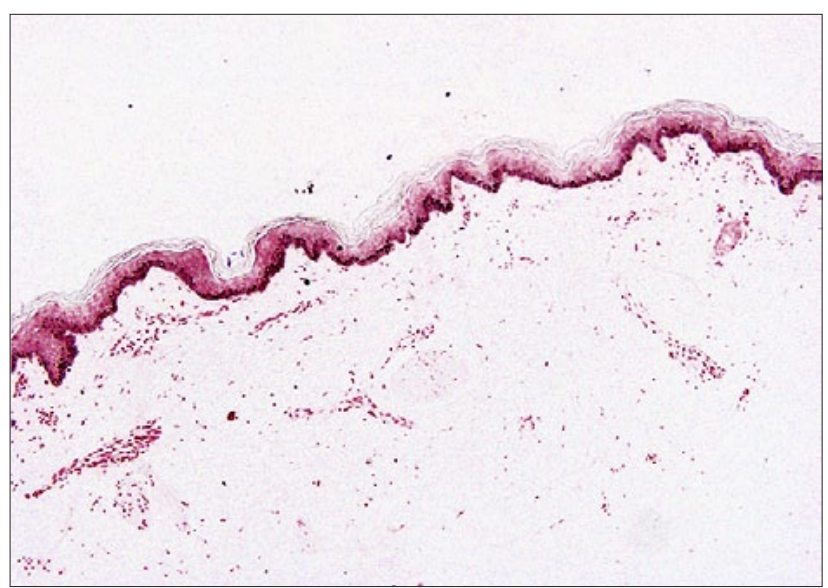

Figure 9. Normal-appearing skin biopsy: Fontana-Masson stained section shows normal melanin pigment of the biopsied normal-appearing skin compared with the reduction of the melanin granules in the basal cell layer of adjacent hypopigmented skin lesion in Figure 8 (Fontana-Masson, 10x). 
Table 2: Regimentation at the end of active treatment (week 14) and at the end of follow-up period (week 38)

\begin{tabular}{llll} 
Total repigmentation & UVB+ Anti acne $(\mathbf{n}=\mathbf{1 5})$ & UVB $(\mathbf{n}=14)$ & P-Value \\
\hline At week 14 & $10(66 \%)$ & $9(64 \%)$ & NS \\
At week 38 & $2(13 \%)$ & $2(14 \%)$ & NS
\end{tabular}

\section{Results}

Thirty-five patients met our inclusion criteria for diagnosis of PMH. Six patients were lost in follow-up and excluded from the analysis. The remaining 29 patients $(7$ men and 22 women) completed all the investigations and treatment course (active treatment and follow-up). Table 1 shows the demographic and clinical criteria of studied patients. All patients were Egyptian of Arabic descent with no racial mixing. The mean age of onset was $20.7 \pm 9.1$ years (range between 16 and 37 years). The mean duration of the disease was $7 \pm 2.1$ years. The back (especially lumbar and lumbosacral areas) was the most common site of involvement followed by abdomen, buttocks, chest, and thighs (Figures 1-3). All lesions fulfill the criteria of PMH with normal sensation and negative $\mathrm{KOH}$ mounts of skin scrapings for Malassezia furfur. Under Wood's lamp examination, hypopigmented lesions demonstrated a follicular pink fluorescence in two patients only.

Normal and lesional skin biopsies were taken for comparison in all 29 patients. Hematoxylin and eosin stained sections showed no significant difference between lesional and normal skin, except in five patients who showed subtle decrease in pigment intensity in lesional skin (Figures 4, 5). There was no spongiosis, inflammatory infiltrate, or epidermotropism in any case. S100 staining did not detect significant differences in the number of melanocytes in lesional and normal-appearing skin (Figures 6, 7). Fontana-Masson stained sections showed overall reduction of melanin granules in the basal cell layer of lesional skin compared to normal adjacent normal-appearing skin (Figures 8, 9). Sections stained with PAS did not show any spores or hyphae in the stratum corneum or pilosebaceous ducts.

The external dermatologist scored the bcUVB-treated group slightly higher than the nbUVb-treated group. Complete or nearly complete repigmentation was reported in 10 patients in the bcUVB-treated group compared to 9 patients in the nbUVb-treated group with no significant differences between both treatment groups after 14 weeks of treatment ( $\mathrm{P}>0.05$ ). At the end of follow-up (at 38 weeks), only two patients in the nbUVb-treated group and two patients in the bcUVB-treated group retained the pigmentation acquired by treatment, and the remaining patients (seven in the nbUVb group and eight in the bcUVB group) returned to baseline color with loss of pigmentation acquired by treatment.

\section{Discussion}

While several reports have documented progressive macular hypomelanosis of the trunk over the past two decades [1-5], it is not a well-recognized entity in clinical practice in many parts of the world, possibly because the initial publications on this subject were not in English. Multiple synonyms, including PMH [1], progressive macular confluent hypomelanosis [3], cutis trunci variata [12], progressive and confluent hypomelanosis of the melanodermic metis [2] nummular and confluent hypomelanosis of the trunk [13], and idiopathic multiple large macules [14] add to the confusion. Similarly, Lernia and Ricci [15] and Relyveld et al [6] concluded that the condition described by Zaynoun and coworkers [16] as extensive pityriasis alba cannot be differentiated from progressive and extensive hypomelanosis described by Guillet et al [1,2]. When occurring in dark-skinned individuals, $\mathrm{PMH}$ may be misdiagnosed as hypopigmentation associated with pityriasis versicolor, despite exclusion of Malassezia furfur. In areas where leprosy is endemic, tuberculoid or borderline tuberculoid leprosy may be considered, but there is no sensory impairment, no textural change, and no histologic evidence of granulomas in lesions of PMH. Hypopigmented mycosis fungoides differs from $\mathrm{PMH}$ in its asymmetrical distribution, poikiloderma, and textural changes [1-4].

Progressive macular hypomelanosis is a disease of unknown etiology. Several theories have been proposed regarding its etiology, including that of its being a genodermatosis [1, 2], or that it is related to P. acnes [5]. Westerhoff, Relyveld, and others published a study in 2004 demonstrating a relationship between the hypopigmented macules and $P$. acnes [5]. Their study included eight patients with biopsy specimens taken of normal and affected skin, and the presence of bacteria was determined by examining fluorescent and non-fluorescent hair follicles using a Wood's lamp and by anaerobic culture. Affected skin fluoresced on Wood's lamp evaluation in all eight patients and culture showed evidence of $P$. acnes on specimens in all but one patient. Normal skin specimens did not show evidence of any bacteria [5].

Successful treatment of PMH has eluded practitioners. Topical steroids and anti-fungals were generally unsuccessful in many of our patients, similar to the findings in other reports. If Westerhoff et al are correct about $P$. acnes playing a role, specifically that $P$. acnes inhibits melanogenesis, then antibiotics such as topical clindamycin or the oral tetracyclines should, in theory, be effective. In 2006 [10], Relyveld 
and others (including Westerhoff) reported that UVA irradiation in combination with benzoyl peroxide $5 \%$ hydrogel in the morning and clindamycin 1\% lotion at night for 14 weeks was more effective than UVA irradiation plus topical steroid for repigmentation in patients with PMH [10]. However, they limited the post-treatment follow-up period to 12 weeks only with no long-term follow-up data.

To our knowledge, there is no available data about the prevalence of $\mathrm{PMH}$ in Egypt. We think that it is not a rare condition in clinical practice, but commonly misdiagnosed and under-reported by treating physicians. In our hospital, the total number of patients with $\mathrm{PMH}$ was greater than the number recruited for the study, because several were excluded due to the refusal of a skin biopsy or due to the presence of unrelated concomitant diseases.

We found that $\mathrm{PMH}$ is a disorder of adolescents and young adults with strong female preponderance $(76 \%)$, as previously reported [1-5]. The age of our patients ranged between 16 and 37 years with no cases reported in the elderly, as noted in other studies. This finding supported that $\mathrm{PMH}$ is not a progressive disorder as expected from the nomenclature of the disease. Therefore, the term "progressive" in PMH may be erroneous because the condition does not progress in most patients and regresses spontaneously after many years, as reported by other authors [4].

We could not identify any definite causative factor(s). Exposure to natural sunlight may enhance the visual demarcation between lesional and nonlesional skin, indicating that $\mathrm{PMH}$ lesions tan poorly as reported in a study in Singapore [4]. In contrast to this observation, temporary repigmentation after natural sun exposure or narrowband UVB has been reported in other studies, including our study [7-9].

All of our patients were Egyptians with no parental consanguinity or racial mixing. Thus, it is clear that PMH can occur in any race, and not only in mixed Negroid subjects as reported previously by Guillet et al [1].

In this study, histological examination demonstrated decreased melanization of the basal layer in lesions of PMH as compared to normal skin. This difference was illustrated with Fontana-Masson stain. S100 staining did not detect significant differences in the number of melanocytes in lesional and normal-appearing skin. There was no spongiosis, inflammatory infiltrate, or epidermotropism in any case. Our results were consistent with previous studies [1-5].

Histologic evaluation is sometimes important to exclude hypopigmented mycosis fungoides, vitiligo, and postinflammatory pigmentary changes. The observation that there was no decrease in numbers of melanocytes but only a decrease in melanin content suggests that there is probably a functional defect in pigmentation or a problem in melanin distribution. Electron microscopic studies [4, 17] revealed that hypopigmentation in PMH seems to be the result of an altered mela- nogenesis based on a decrease in melanin formation and a change in the distribution of melanosomes. In lesional skin of $\mathrm{PMH}$ patients less melanized aggregated melanosomes instead of single, mature melanosomes are transferred from melanocytes to keratinocytes $[4,17]$. This results in a decrease of epidermal melanin [4, 17]. A hypothetical factor produced by $P$. acnes may be responsible for abnormal melaogenesis, as suggested by the authors who claimed that this organism may have a role in pathogenesis of PMH [5, 17]. The clinical observations that PMH fades over time and that it is not found in the elderly suggest that the inhibitory effect on melanogenesis in the lesional skin of those patients is not permanent $[4,5]$.

$\mathrm{PMH}$ is known to be recalcitrant to treatment. Few reports claimed the effectiveness of regimentation after narrow band UVB [7, 8], combination of sunlight and oral tetracycline (case report) [9], combination of UVA1 plus topical clindamycin and benzoyl peroxide (45 patients) [10], or combination of topical benzoyl peroxide and oral doxycycline (case report) [11]. However, long-term follow-up data are lacking in all studies, and the maximum follow-up duration in most studies was three months.

In our work, we divided the patients randomly into two groups: the first group received narrow-band UVB treatment and the other group received the same regimen of the first group (narrow-band UVB treatment) plus daily topical clindamycin solution $1 \%$ in the morning and benzoyl peroxide gel $5 \%$ in the evening. Active treatment was continued for 14 weeks followed by a follow-up treatment-free period of 24 weeks, which is nearly double the follow-up period in the Relyveld et al study [10]. At the end of active treatment period, total repigmentation was obtained in 10/15 patients in the bcUVB group compared to $8 / 14$ in the nbUVB group with no significant difference between both groups. These results were nearly similar to Relyveld et al [10] who used a combination of UVA1 plus topical measures against P.acne (clindamycin and benzoyl peroxide). However, our results were dramatically different from the last authors at the end of follow-up period. Relyveld et al [10] reported that most of their patients retained the pigmentation at the end of a follow-up period of 12 weeks. In our work, we extended the follow-up period to 24 weeks and we found that most of our patients in both groups lost the pigmentation acquired by treatment in both groups. These results do not support the pathogenic role of $P$. acnes in PMH as claimed by Westerhoff and his team. However, regrowth of $P$. acnes may provide an explanation for loss of pigmentation acquired in treatment. 


\section{Conclusion}

Progressive macular hypomelanosis is a disease of unclear etiology that is often misdiagnosed and difficult to treat. This study documented the clinicopathological features of $\mathrm{PMH}$ among Egyptian patients. No permanently effective treatment is available. Further studies on a larger number of patients are needed to prove or disprove the pathogenic role of P. acnes in PMH and to search for other cause(s) or other effective treatments for this disfiguring disease.

\section{References}

1. Guillet G, Helenon R, Gauthier Y, Surleve-Bazeille JE, Plantin P, Sassolas B. Progressive macular hypomelanosis of the trunk: primary acquired hypopigmentation. J Cutan Pathol 1988;15(5):286-9.

2. Guillet G, Halenon R, Guillet MH, Gauthier Y, Menard N. [Progressive and confluent hypomelanosis of the melanodermic metis]. Ann Dermatol Venereol 1992;119(1):19-24.

3. Lesueur A, Gracia-Granel V, Halenon R, Cales-Quist D. [Progressive macular confluent hypomelanosis in mixed ethnic melanodermic subjects: an epidemiologic study of 511 patients]. Ann Dermatol Venereol 1994;121(12):880-3.

4. Kumarasinghe SPW, Tan SH, Thng S, Thamboo TP, Liang S, Lee YS. Progressive macular hypomelanosis in Singapore: a clinicopathological study. Int J Dermatol 2006;45(6):737-42.

5. Westerhof W, Relyveld GN, Kingswijk MM, de Man P, Menke HE. Propionibacterium acnes and the pathogenesis of progressive macular hypomelanosis. Arch Dermatol 2004;140(2):210-4.

6. Relyveld G, Menke H, Westerhof W. Progressive and extensive hypomelanosis and extensive pityriasis alba: same disease, different names? (letter). J Eur Acad Dermatol Venereol 2006;20(10):13631364.

7. Hwang SW, Hong SK, Kim SH, et al. Progressive macular hypomelanosis in Koreans: a clinicopathologic study. Ann Dermatol 2009;21(3):261-7.

8. Chung YL, Goo B, Chung WS, Lee GS, Hann SK. A case of progressive macular hypomelanosis treated with narrow-band UVB (letter). J Eur Acad Dermatol Venereol 2007;21(7):1007-9.

9. Perman M, Sheth P, Lucky AW. Progressive macular hypomelanosis in a 16-year old. Pediatr Dermatol 2008;25(1):63-5.

10. Relyveld GN, Kingswijk M, Reitsma JB, Menke HE, Bos JD, Westerhof W. Benzoyl peroxide/clindamycin/UVA is more effective than fluticasone/UVA in progressive macular hypomelanosis: a randomized study. J Am Acad Dermatol 2006;55(5):836-43.

11. Tierney E, Hamzavi I. Progressive macular hypomelanosis in a young African-American woman: case report and review of the literature. J Am Acad Dermatol 2007;56:2, (Supplement 2) AB146.

12. Borelli D. [Cutis "trunci variata." A new genetic dermatosis]. Med Cutan Ibero Lat Am 1987;15(4):317-19.

13. Menke HE, Doornweerd S, Zaal J, et al. Acquired nummular and confluent hypomelanosis of the trunk. Paper presented at: Second Annual Meeting of the European Society for Pigment Cell Research; June 18-21, 1989; Uppsala, Sweden.
14. Sober AJ, Fitzpatrick TB. Yearbook of Dermatology. St Louis, MO: Mosby-Year Book, 1996:416-7.

15. Di Lernia V, Ricci C. Progressive and extensive hypomelanosis and extensive pityriasis alba: same disease, different names? J Eur Acad Dermatol Venereol 2005;19(3):370-2.

16. Zaynoun ST, Aftimos BG, Tenekjian KK, Bahuth N, Kurban AK. Extensive pityriasis alba: a histological histochemical and ultrastructural study. Br J Dermatol 1983;108(1):83-90.

17. Relyveld G, Dingemans KP, Menke HE, Bos JD, Westerhof W. Ultrastructural findings in progressive macular hypomelanosis indicate decreased melanin production. J Eur Acad Dermatol Venereol 2008;22(5):568-74. 